\title{
INFLUÊNCIA DA LUMINÂNCIA DO NEGATOSCÓPIO NA DETECTABILIDADE DE FIBRAS E MICROCALCIFICAÇÕES, DETERMINADA POR MEIO DE OBJETOS SIMULADOS*
}

\author{
Regina Bitelli Medeiros' ${ }^{1}$, Fátima Faloppa Rodrigues Alves ${ }^{2}$, Eny Moreira Ruberti Filha ${ }^{3}$, Flora \\ Fingerman ${ }^{4}$, Adriana Helena Padovan ${ }^{4}$, Simone Elias ${ }^{5}$, Daniela Stanzani ${ }^{4}$, Claudio Kemp ${ }^{6}$
}

Resumo Considerando a natureza sutil dos achados mamográficos, é importante conhecermos como a detectabilidade de pequenos objetos, como fibras e microcalcificações, normalmente presentes em imagens mamográficas, pode ser influenciada pela intensidade luminosa do negatoscópio utilizado nas leituras. Nosso objetivo foi verificar como a luminância pode alterar a detectabilidade de objetos simulados. Foram feitas sete imagens com diferentes intensidades de exposição em duas condições técnicas usando um simulador que permite avaliações estatísticas. Os resultados foram computados usando uma escala de cinco níveis de confiança (100 se o especialista tem certeza da presença do objeto; 75 se provável a presença do objeto; 50 se incerta; 25 se improvável; zero se definitivamente ausente). As imagens foram interpretadas por especialistas que utilizaram negatoscópios "padrões" e específicos para imagens mamográficas. As detectabilidades foram estatisticamente comparadas entre si para cada tipo de negatoscópio por meio do teste do qui-quadrado de Pearson $(p<0,05)$. A detectabilidade variou de 0,79 a 0,87 . Pudemos concluir que a detectabilidade dos objetos, determinada por meio de objetos simulados, é superior para os negatoscópios específicos para mamografias quando comparada aos negatoscópios "padrões" amplamente utilizados na radiologia geral. Unitermos: Negatoscópio; Controle de qualidade; Mamografia; Luminância; Lâmpada fluorescente.

\begin{abstract}
The influence of viewing boxes luminance on the detectability of fibers and microcalcifications using simulated objects.

Considering the subtle findings observed in mammography images, it is relevant to know how detectability of fibers and microcalcifications can be influenced by the luminance of the viewing boxes. Our goal was to determine the influence of the luminance on the detectability of tiny simulated objects. We obtained seven images with different exposure levels using a phantom that allows statistical analysis. The results were computed using a five point scale to report the confidence levels (100 if the evaluator was sure that the object is present; 75 if likely; 50 if uncertain; 25 if not likely; zero if definitely absent). The images were analyzed by specialists using a "standard" and a specific viewing box for mammography. The detectability was compared using the Pearson chi-square test $(p<0.05)$. The values varied from 0.79 to 0.87 . We concluded that the detectability determined from simulated objects through readings on the specific viewing boxes is higher when compared with the readings using standard viewing boxes.

Key words: View box; Quality control; Mammography; Luminance; Illuminators; Fluorescent tube.
\end{abstract}

\section{INTRODUÇÃO}

Considerando a natureza sutil dos achados mamográficos, é importante co-

* Trabalho realizado na Escola Paulista de Medicina - Universidade Federal de São Paulo (Unifesp-EPM), São Paulo, SP.

1. Professora Adjunta Doutora, Chefe da Coordenadoria de Física e Higiene das Radiações da EPM-Unifesp.

2. Física da Unifesp, Mestre em Ciências Radiológicas.

3. Física Especializanda da Unifesp.

4. Médicas Radiologistas do Departamento de Diagnóstico por Imagem da EPM-Unifesp.

5. Médica Pós-graduanda do Setor de Mastologia do Departamento de Ginecologia da EPM-Unifesp.

6. Professor Adjunto Doutor, Chefe do Setor de Mamografia do Departamento de Diagnóstico por Imagem da EPM-Unifesp.

Endereço para correspondência: Dra. Regina Bitelli Medeiros. Rua Botucatu, 659, Vila Clementino. São Paulo, SP, 04023-062. E-mail: rbitelli.ddi@epm.br

Recebido para publicação em 4/3/2002. Aceito, após revisão, em 10/10/2002. nhecermos as probabilidades de detectabilidade de objetos rotineiramente presentes em imagens mamográficas, como fibras e microcalcificações, e como podem ser influenciadas pelas condições de exposição e visibilidade das imagens ${ }^{(\mathbf{1 , 2})}$.

A luminância do negatoscópio utilizado pelo especialista na avaliação da imagem constitui um dos parâmetros que deve ser controlado, entre outros tantos definidos no programa de garantia da qualidade de um serviço de radiologia, previsto pela Portaria 453/98, do Ministério da Saúde ${ }^{(3)}$. A luminância pode influenciar a visibilidade de objetos sutis e a decisão dos especialistas sobre a malignidade do achado de sistemas de imagens. Mudanças no padrão da imagem radiológica e/ou mesmo na exposição ou densidade óptica do filme, eventualmente consideradas irrelevantes, podem mascarar um achado clínico importante caso a luminância do negatoscópio não esteja dentro dos padrões estabelecidos pela legislação vigente no Brasil (3.500 nit para mamografia $)^{(3)}$. Os padrões de aceitação internacionais especificam em 1.500 nit o desempenho mínimo para negatoscópios utilizados na radiologia geral ("padrão") e de 3.000 a 3.500 nit para negatoscópios específicos para leituras de imagens mamográficas. O desempenho requerido para luminosidade ambiental é de 50 lux. Quanto à uniformidade da luminância, é aceitável uma variação de até $15 \%{ }^{(4,5)}$. 
A intensidade de luz de um negatoscópio depende principalmente da temperatura ambiente, horas de funcionamento das lâmpadas fluorescentes, potência da lâmpada, tipo e espessura do material utilizado na fabricação do painel frontal, e estado de conservação do negatoscópio ${ }^{(6)}$. Foi demonstrado que negatoscópios "padrões" em bom estado e com lâmpadas novas são capazes de proporcionar no mínimo 1.650 $\mathrm{cd} / \mathrm{m}^{2}$, após aquecidos, podendo nas mesmas condições proporcionar luminância de até $2.300 \mathrm{~cd} / \mathrm{m}^{2}$, dependendo do tipo do painel frontal, em geral tela acrílica, e do fabricante das lâmpadas ${ }^{(7)}$.

Por meio de estudos anteriores detectamos um decréscimo da detectabilidade de objetos simulados nas imagens com intensidade de exposição ou densidades ópticas maiores, responsável por uma diminuição da sensibilidade ou acuidade do diagnóstico nessas imagens ${ }^{(\mathbf{8})}$. Resta-nos saber se a seleção do negatoscópio, entre o utilizado como "padrão" na radiologia e o comercializado como específico para leitura de imagens mamográficas, pode minimizar a dificuldade do especialista em detectar estruturas de natureza sutil, eventualmente presentes nessas imagens.

Nosso objetivo foi verificar a influência da luminância do negatoscópio na detectabilidade de fibras e microcalcificações presentes em imagens mamográficas simuladas com diferentes densidades ópticas, efetuadas sob controle de qualidade de processamento, segundo o protocolo do American College of Radiology (ACR) ${ }^{(4)}$.

\section{MATERIAL E MÉTODO}

Mamógrafo Philips modelo MD 4000; sistema tela/filme Min-R 2000/Min-R 2000 - Kodak; químico RPX-OMAT Kodak; processadora modelo M35 dedicada - Kodak; densitômetro modelo 380 XRite; "mammographic accreditation phantom" modelo 156 Gammex/RMI (ACR); simulador estatístico model 18-209-Alvim - TRM; fotômetro Luminance meter Minolta modelo LS-100; negatoscópios “padrões" e específicos.

Efetuamos imagens do simulador de acrílico de 4,5 cm (ACR), utilizado rotineiramente no programa de garantia da qualidade, no modo de controle automático da exposição ("automatic exposure control" - AEC) a 28 kVp, em condições controladas de processamento. Utilizando o densitômetro, determinamos a densidade óptica na região central dessas imagens para verificar se esta densidade encontrava-se dentro do intervalo calibrado pelo fabricante do mamógrafo $(1,4$ a 1,8), para as condições de processamento e sistema telafilme utilizados. Utilizando o simulador Alvim (Figura 1B), efetuamos exposições que variaram $\pm 30 \%$, em torno deste ponto, para duas condições técnicas $(24 \mathrm{kV}$ e $28 \mathrm{kV}$ ), totalizando 56 imagens em sete níveis de densidade óptica em cada técnica. A cada quatro imagens, efetuadas para cada intensidade de exposição, modificamos randomicamente a posição das microcalcificações e fibras de diferentes dimensões que compõem o simulador.

As imagens foram interpretadas por três especialistas que utilizaram negatoscópios de sua rotina de trabalho, dos tipos "padrão" e específico para mamografia. Medimos a luminância desses negatoscópios por meio do fotômetro e determinamos, para cada um deles, a média e o coeficiente de variação das medidas originadas de cinco regiões, tais como: lateral direita superior e inferior, lateral esquerda superior e inferior e centro.

Os especialistas utilizaram uma escala de cinco níveis de confiança, atribuindo a pontuação 100 (quando tinham certeza da presença do objeto), 75 (quando julgavam provável a presença do objeto), 50 (quando julgavam incerta a presença do objeto), 25 (quando julgavam improvável a presença do objeto) e 0 (quando julgavam o objeto definitivamente ausente), conforme sugestão do fabricante do simulador. Computamos as pontuações atribuídas pelos especialistas, independentemente para cada negatoscópio, a fim de determinarmos a detectabilidade dos objetos, calculada a partir da expressão:

$$
\begin{gathered}
\text { Pdet }=[\mathrm{P}(\mathrm{VP})+\mathrm{P}(\mathrm{VN})] /[\mathrm{P}(\mathrm{VP})+ \\
+\mathrm{P}(\mathrm{VN})+\mathrm{P}(\mathrm{FP})+\mathrm{P}(\mathrm{FN})],
\end{gathered}
$$

onde:

$\mathrm{P}(\mathrm{VP})$, proporção de resultados positivos quando há objetos presentes (equivalente à probabilidade de reconhecer a patologia): sensibilidade;

$\mathrm{P}(\mathrm{VN})$, proporção de resultados negativos quando não há objetos presentes (equivalente à probabilidade de reconhecer a "normalidade"): sensitividade;

$\mathrm{P}(\mathrm{FP})$, proporção de resultados positivos na ausência de objetos;

$\mathrm{P}(\mathrm{FN})$, proporção de resultados negativos quando há objetos realmente presentes.

Estudamos a influência da luminância do negatoscópio na detectabilidade de fibras e de microcalcificações para cada um dos especialistas, computando todas as respostas que indicavam a presença desses objetos, independentemente da confiabilidade dessas respostas, atribuindo o valor 1 a cada achado, com a finalidade de conhecermos as características estatísticas relacionadas à detecção de cada uma dessas duas estruturas.

As detectabilidades clínicas dos objetos foram estatisticamente comparadas entre si, para cada tipo de negatoscópio, por intermédio do teste do qui-quadrado de Pearson $(\mathrm{p}<0,05)$.

\section{RESULTADOS}

Por meio das imagens efetuadas com o simulador ACR (Figura 1A), pudemos nos certificar da invariabilidade das condições de processamento e da qualidade do sistema, uma vez que a leitura da densidade óptica efetuada no centro do simulador manteve-se em 1,7 durante todo o estudo.

Os resultados da luminância dos negatoscópios utilizados pelos especialistas estão apresentados na Tabela 1. A luminosidade ambiental durante as leituras das imagens simuladas foi inferior a 30 lux. Os especialistas utilizaram "máscara” sobre o negatoscópio para impedir a difusão de luz devido a áreas descobertas, e ainda lupa durante as leituras, conforme condições de rotina. O coeficiente de variação da luminância entre os negatoscópios utilizados foi de $17,7 \%$ para os denominados "padrão" e de $12,3 \%$ para os específicos para leituras mamográficas.

A Tabela 2 mostra os resultados para microcalcificações e fibras para os três observadores em função das densidades ópticas utilizadas, calculadas independentemente dos graus de confiança nos achados. Com base nesses dados, a análise estatística mostrou que em geral a detectabilidade de fibras é superior à de microcal- 


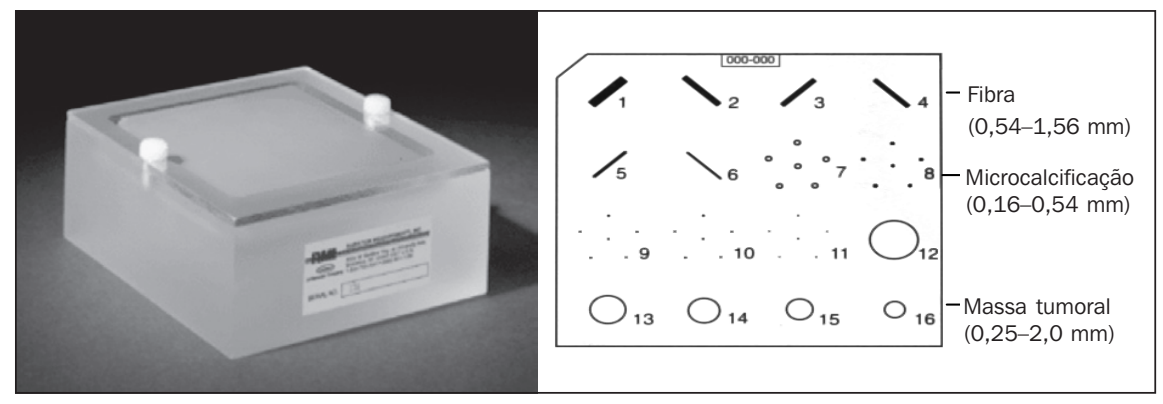

A

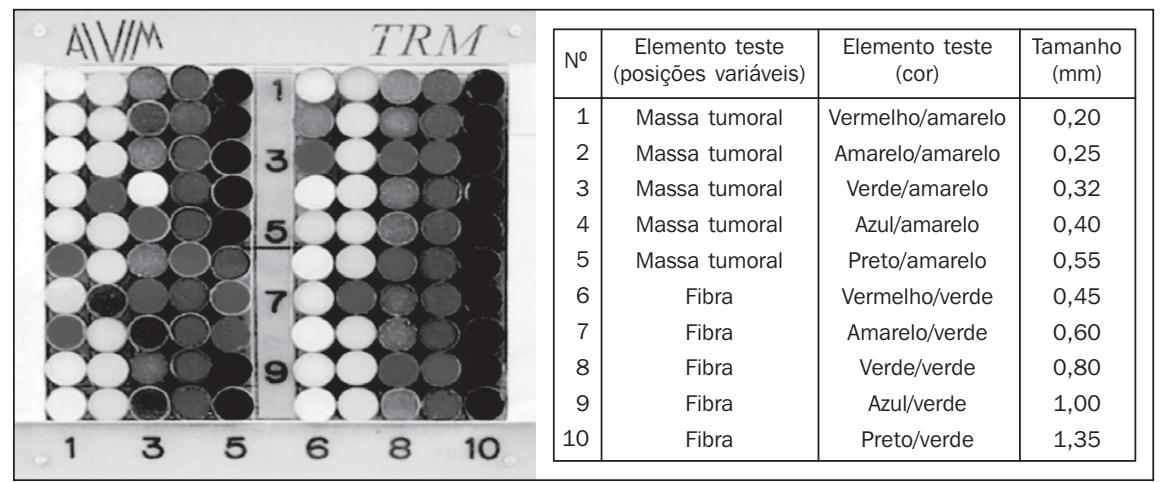

B

Figura 1. Características dos tamanhos e posições dos objetos existentes nos simuladores radiográficos de mama. A: Modelo 156 Gammex/RMI. B: Modelo Alvim.
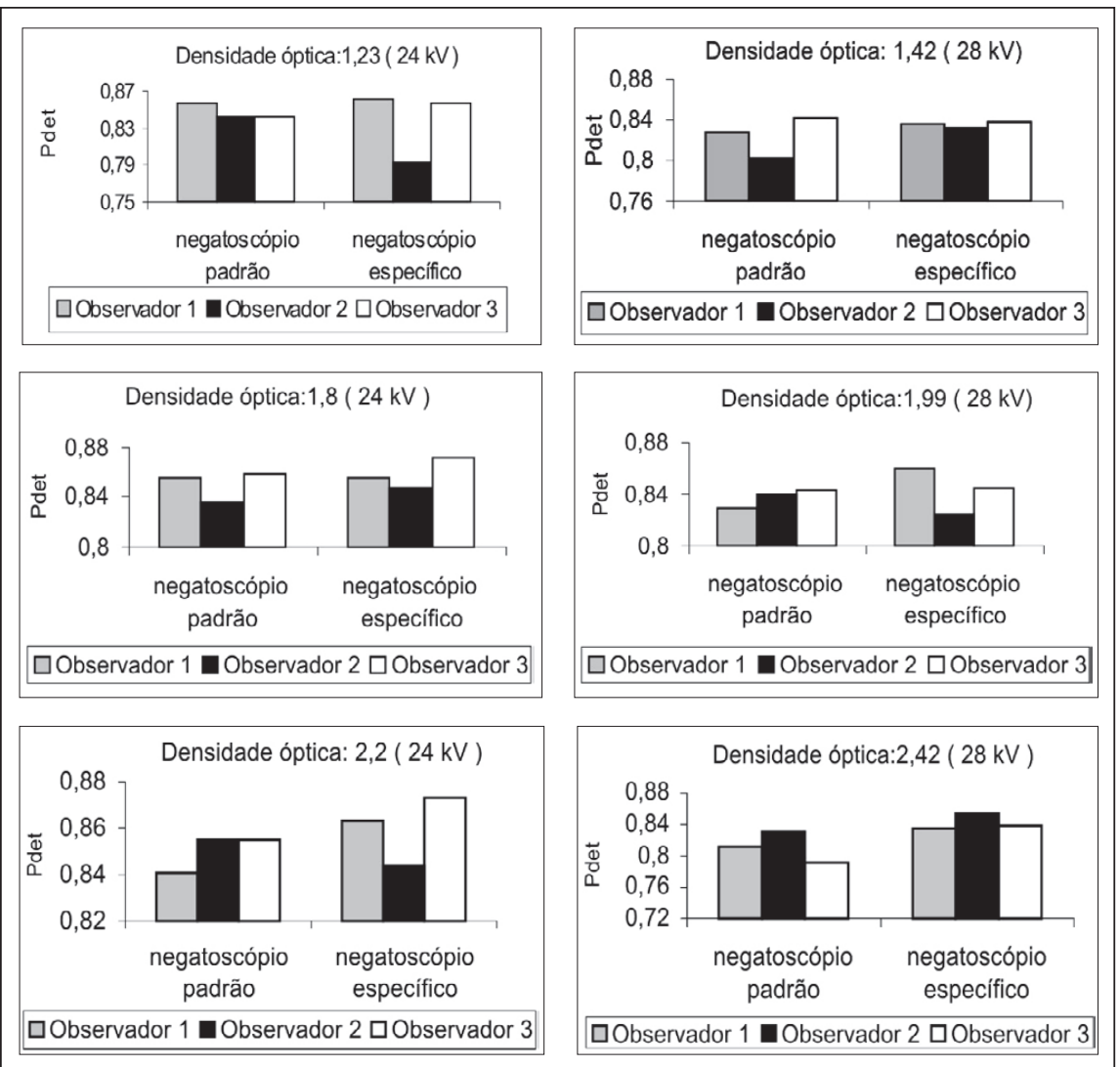

Figura 2. Detectabilidade clínica dos três observadores, obtida para os dois tipos de negatoscópio em diferentes condições técnicas. cificações, para as imagens interpretadas em ambos os negatoscópios. A Figura 2 mostra a detectabilidade dos observadores considerando os diferentes níveis de confiança atribuídos às imagens de densidades ópticas referentes ao ponto de calibração, $30 \%$ superior e $30 \%$ inferior a esse ponto, avaliadas nos dois tipos de negatoscópios para 24 e 28 kV. Como exemplo, a Figura 3 mostra a detectabilidade das estruturas obtidas para cada observador, em ambos os negatoscópios.

\section{DISCUSSÃO}

Pudemos observar diferenças individuais entre os observadores no julgamento da presença dos objetos. Essas diferenças podem ser evidenciadas quando a subjetividade é quantificada pelo grau de confiabilidade na detecção dos objetos presentes (VP e VN) e ausentes (FP e FN) ${ }^{(\mathbf{1})}$.

Constatamos um maior número de diferenças obtidas na técnica de $28 \mathrm{kV}$ para o negatoscópio específico, sugerindo uma

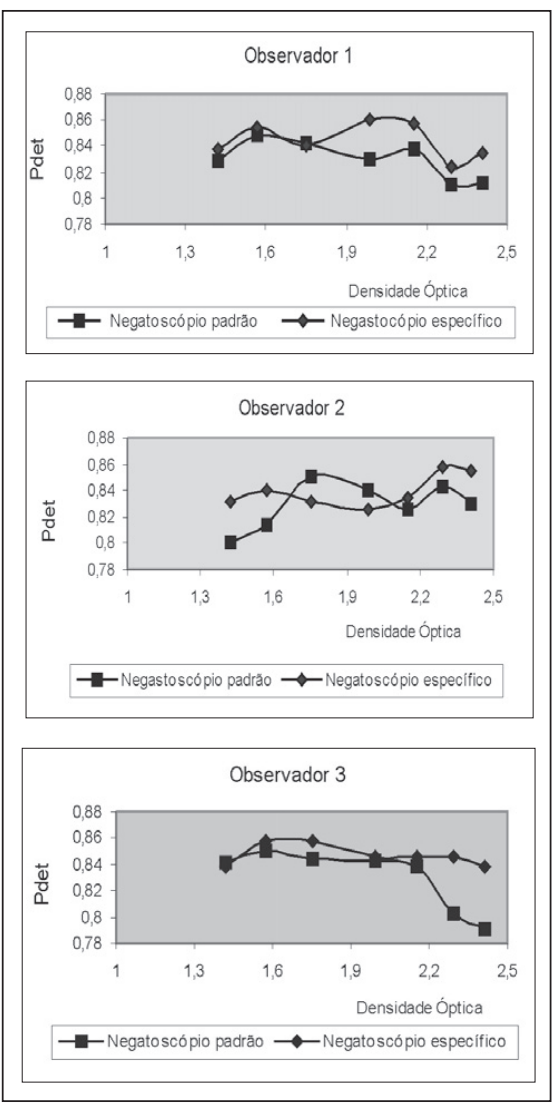

Figura 3. Detectabilidade clínica de cada observador em ambos os negatoscópios, para $28 \mathrm{kVp}$ (AEC). 
Tabela 1 Valores de média, desvio e coeficiente de variação da luminância dos negatoscópios utilizados nas leituras das imagens.

\begin{tabular}{|c|c|c|l|}
\hline Negatoscópio & Luminância $\left(\mathrm{cd} / \mathrm{m}^{2}\right)$ & Coeficiente de variação & Uso \\
\hline 1 & $1.531,90 \pm 605,59$ & 0,39 & \\
2 & $1.791,70 \pm 710,52$ & 0,39 & Radiologia geral \\
3 & $1.733,90 \pm 633,45$ & 0,36 & ("padrão" \\
4 & $2.297,20 \pm 496,94$ & 0,22 & \\
\hline 5 & $3.147,51 \pm 506,23$ & 0,16 & Mamografia \\
6 & $3.404,40 \pm 477,83$ & 0,14 & ("específico") \\
7 & $2.664,60 \pm 356,78$ & 0,13 & \\
8 & $2.669,00 \pm 465,58$ & 0,17 & \\
\hline
\end{tabular}

Tabela 2 Resultados obtidos para microcalcificações e fibras, para os três observadores, em função da densidade óptica, independentemente da confiabilidade dos achados.

\begin{tabular}{|c|c|c|c|c|c|c|c|c|c|c|c|c|}
\hline \multirow[b]{4}{*}{ DO } & \multicolumn{12}{|c|}{ NEGATOSCÓPIO PADRÃO (24kV) } \\
\hline & \multicolumn{6}{|c|}{ Microcalcificação } & \multicolumn{6}{|c|}{ Fibra } \\
\hline & \multicolumn{2}{|c|}{$1^{\circ} \mathrm{Obs}}$. & \multicolumn{2}{|c|}{$2^{\circ}$ Obs. } & \multicolumn{2}{|c|}{$3^{\circ}$ Obs. } & \multicolumn{2}{|c|}{$1^{\circ} \mathrm{Obs}}$. & \multicolumn{2}{|c|}{$2^{\circ}$ Obs. } & \multicolumn{2}{|c|}{$3^{\circ} \mathrm{Obs}$. } \\
\hline & $\mathrm{FN}$ & FP & $\mathrm{FN}$ & FP & FN & FP & FN & FP & $\mathrm{FN}$ & FP & $\mathrm{FN}$ & FP \\
\hline 1,23 & 0,34 & 0 & 0,15 & 0,34 & 0,27 & 0,08 & 0,13 & 0,02 & 0,05 & 0,31 & 0,18 & 0,02 \\
\hline 1,41 & 0,33 & 0 & 0,13 & 0,36 & 0,29 & 0,18 & 0,14 & 0,08 & 0,03 & 0,41 & 0,16 & 0,04 \\
\hline 1,56 & 0,34 & 0 & 0,08 & 0,23 & 0,25 & 0,16 & 0,13 & 0,02 & 0,04 & 0,37 & 0,12 & 0,04 \\
\hline 1,80 & 0,33 & 0 & 0,13 & 0,31 & 0,24 & 0,17 & 0,11 & 0,03 & 0,03 & 0,52 & 0,17 & 0,04 \\
\hline 1,97 & 0,35 & 0 & 0,13 & 0,32 & 0,25 & 0,16 & 0,10 & 0,02 & 0,04 & 0,43 & 0,15 & 0,01 \\
\hline 2,10 & 0,35 & 0 & 0,12 & 0,21 & 0,25 & 0,15 & 0,15 & 0,04 & 0,05 & 0,28 & 0,18 & 0,03 \\
\hline 2,20 & 0,33 & 0 & 0,12 & 0,21 & 0,29 & 0,18 & 0,21 & 0,03 & 0,03 & 0,32 & 0,17 & 0,02 \\
\hline DO & \multicolumn{12}{|c|}{ NEGATOSCÓPIO ESPECÍFICO (24kV) } \\
\hline 1,23 & 0,31 & 0 & 0,14 & 0,29 & 0,28 & 0 & 0,13 & 0,02 & 0,07 & 0,41 & 0,21 & 0,02 \\
\hline 1,41 & 0,32 & 0 & 0,17 & 0,16 & 0,25 & 0,06 & 0,15 & 0,08 & 0,02 & 0,44 & 0,16 & 0,09 \\
\hline 1,50 & 0,32 & 0 & 0,11 & 0,16 & 0,27 & 0,06 & 0,12 & 0,03 & 0,05 & 0,29 & 0,08 & 0,05 \\
\hline 1,80 & 0,31 & 0,01 & 0,17 & 0,23 & 0,26 & 0,02 & 0,14 & 0,01 & 0,07 & 0,29 & 0,15 & 0,03 \\
\hline 1,97 & 0,32 & 0 & 0,13 & 0,19 & 0,23 & 0,05 & 0,12 & 0,05 & 0,14 & 0,19 & 0,10 & 0,02 \\
\hline 2,10 & 0,33 & 0 & 0,18 & 0,11 & 0,26 & 0,02 & 0,17 & 0,02 & 0,09 & 0,18 & 0,14 & 0,06 \\
\hline \multirow[t]{4}{*}{2,20} & 0,31 & 0 & 0,17 & 0,14 & 0,27 & 0,04 & 0,11 & 0,04 & 0,06 & 0,23 & 0,12 & 0,03 \\
\hline & \multicolumn{12}{|c|}{ NEGATOSCÓPIO PADRÃO (28kV) } \\
\hline & \multicolumn{6}{|c|}{ Microcalcificação } & \multicolumn{6}{|c|}{ Fibra } \\
\hline & \multicolumn{2}{|c|}{$1^{\circ} \mathrm{Obs}}$. & \multicolumn{2}{|c|}{$2^{\circ}$ Obs. } & \multicolumn{2}{|c|}{$3^{\circ}$ Obs. } & \multicolumn{2}{|c|}{$1^{\circ} \mathrm{Obs}}$. & \multicolumn{2}{|c|}{$2^{\circ}$ Obs. } & \multicolumn{2}{|c|}{$3^{\circ}$ Obs. } \\
\hline DO & FN & FP & FN & FP & FN & FP & FN & FP & FN & FP & FN & FP \\
\hline 1,42 & 0,32 & 0 & 0,14 & 0,38 & 0,27 & 0,02 & 0,25 & 0,02 & 0,07 & 0,54 & 0,25 & 0,02 \\
\hline 1,57 & 0,33 & 0,01 & 0,16 & 0,33 & 0,24 & 0 & 0,14 & 0,08 & 0,05 & 0,45 & 0,17 & 0,03 \\
\hline 1,75 & 0,33 & 0 & 0,11 & 0,25 & 0,30 & 0,02 & 0,16 & 0,02 & 0,04 & 0,36 & 0,19 & 0,02 \\
\hline 1,99 & 0,34 & 0 & 0,17 & 0,29 & 0,27 & 0,01 & 0,20 & 0,06 & 0,04 & 0,32 & 0,19 & 0,03 \\
\hline 2,15 & 0,35 & 0 & 0,13 & 0,45 & 0,29 & 0,02 & 0,19 & 0,03 & 0,08 & 0,40 & 0,27 & 0,02 \\
\hline 2,29 & 0,39 & 0 & 0,11 & 0,19 & 0,32 & 0 & 0,30 & 0,01 & 0,08 & 0,34 & 0,30 & 0,03 \\
\hline 2,41 & 0,36 & 0 & 0,19 & 0,28 & 0,31 & 0 & 0,30 & 0,01 & 0,14 & 0,26 & 0,34 & 0 \\
\hline DO & \multicolumn{12}{|c|}{ NEGATOSCÓPIO ESPECÍFICO (28kV) } \\
\hline 1,42 & 0,36 & 0 & 0,18 & 0,16 & 0,22 & 0,05 & 0,22 & 0,03 & 0,13 & 0,22 & 0,22 & 0,10 \\
\hline 1,57 & 0,32 & 0 & 0,21 & 0,18 & 0,26 & 0,10 & 0,15 & 0,06 & 0,09 & 0,38 & 0,15 & 0,05 \\
\hline 1,75 & 0,31 & 0 & 0,13 & 0,20 & 0,29 & 0 & 0,21 & 0,05 & 0,04 & 0,40 & 0,17 & 0,02 \\
\hline 1,99 & 0,33 & 0,02 & 0,18 & 0,29 & 0,29 & 0,01 & 0,15 & 0,04 & 0,05 & 0,42 & 0,19 & 0,06 \\
\hline 2,15 & 0,37 & 0 & 0,16 & 0,20 & 0,27 & 0,11 & 0,16 & 0,04 & 0,09 & 0,34 & 0,19 & 0,03 \\
\hline 2,29 & 0,35 & 0,01 & 0,24 & 0,09 & 0,29 & 0,05 & 0,17 & 0,11 & 0,14 & 0,16 & 0,18 & 0,05 \\
\hline 2,41 & 0,38 & 0 & 0,22 & 0,08 & 0,30 & 0,05 & 0,22 & 0,03 & 0,16 & 0,09 & 0,21 & 0,05 \\
\hline
\end{tabular}

DO, densidade óptica; FN, falso-negativo; FP, falso-positivo. menor influência da luminância no processo de leitura das imagens obtidas com 24 $\mathrm{kV}$, que pode ser explicada pelo contraste superior dessas imagens.

Os resultados referentes à sensibilidade e à especificidade obtidos para os diferentes observadores são compatíveis com o grau de experiência dos observadores. Os falso-positivos e falso-negativos (FP e FN) são superiores para as microcalcificações, indicando uma chance maior de falhas no diagnóstico dessas estruturas quando comparadas às que simulam as massas tumorais (fibras).

O programa de garantia da qualidade na mamografia propõe garantir um padrão de qualidade da imagem capaz de assegurar a detectabilidade de objetos diminutos e identificar, sempre que presentes no tecido mamário, estruturas suspeitas de malignidade. A detectabilidade dessas estruturas é influenciada pelas condições de visibilidade na interpretação das imagens. A não uniformidade da intensidade de luz do negatoscópio e a luminosidade excessiva do ambiente podem mascarar um achado clínico importante ${ }^{(\mathbf{9 , 1 0})}$. A diferença de intensidade luminosa entre áreas de um mesmo negatoscópio e entre os negatoscópios pode influenciar a detectabilidade dos objetos. Considerando o uso da máscara na leitura das imagens (todas as imagens eram posicionadas na região central dos negatoscópios), consideramos satisfatória a uniformidade da luminância entre os negatoscópios de um mesmo tipo. Porém, não podemos deixar de observar que a uniformidade dos negatoscópios do tipo "padrão" não atende os limites de desempenho especificados pelas normas ${ }^{(4)}$. A exemplo de outros pesquisadores, pudemos quantificar a diferença de detectabilidade de calcificações e correlacioná-la à densidade óptica da imagem, evidenciando a perda de detecção para as densidades ópticas maiores ${ }^{(\mathbf{1 0})}$. Este resultado corrobora a necessidade de se manter o mamógrafo calibrado para condições de processamento e sistema tela-filme que gerem densidade óptica no centro do simulador entre 1,4 e 1,8 quando exposto nas condições técnicas utilizadas na aferição (28 kV, AEC, ponto "N").

Não é raro o uso de negatoscópios para leitura de mamografias contendo internamente duas lâmpadas fluorescentes (tipo 
padrão). Estes fornecem, em sua maioria, luminância menor do que $2.000 \mathrm{~cd} / \mathrm{m}^{2}$, valores estes inferiores aos recomendados pelas normas vigentes ${ }^{(\mathbf{3 , 4 , 8})}$.

Com base nos resultados obtidos por alguns pesquisadores ${ }^{\left({ }^{(6)}\right.}$, mesmo um negatoscópio novo padrão não atende à legislação quando se trata de leituras de imagens mamográficas, para as quais são requeridos como padrão de desempenho 3.500 nit $^{(3)}$. Segundo o fabricante de lâmpadas fluorescentes Philips, a depreciação do fluxo luminoso de algumas lâmpadas, decorrente da variação da temperatura, pode atingir $25 \%$ para temperaturas ao redor de $50^{\circ} \mathrm{C}$, valor este possivelmente equivalente à temperatura interna dos negatoscópios. A emissão de luz é afetada por variações na temperatura ambiente, pois estas produzem variações similares na parede do bulbo, que se tornam evidentes com o aumento da temperatura acima de $25^{\circ} \mathrm{C}^{(\mathbf{5})}$. A manutenção do fluxo luminoso é dependente do tipo de lâmpada e potência. A curva de mortalidade das lâmpadas indica que elas começam a falhar mais rapidamente ao atingirem $70 \%$ da vida estimada $^{(5)}$. Para alguns tipos de lâmpadas de fabricação Sylvania, segundo informações técnicas da empresa, após 4.800 horas de funcionamento há uma redução de $13 \%$ da intensidade da luz. Isto significa que qualquer negatoscópio, após um ano de uso contínuo, pode apresentar redução importante na luminância, e aqueles principalmente utilizados para o diagnóstico necessitam de uma avaliação anual para verificação da necessidade de reposição das lâmpadas fluorescentes.

O uso de quatro lâmpadas tem-se propagado e proporciona uma luminância por volta de $2.500 \mathrm{~cd} / \mathrm{m}^{2}$, ainda inferior a 3.500 nit ou $3.500 \mathrm{~cd} / \mathrm{m}^{2}$, recomendada pela Portaria 453 do Ministério da Saúde e Vigilância Sanitária do Estado de São Paulo. O negatoscópio específico para mamo- grafia é o mais adequado para a visibilidade de estruturas pequenas e de baixo contraste, como a que se quer visualizar nas imagens de mamografia. Deve ser capaz de fornecer $3.500 \mathrm{~cd} / \mathrm{m}^{2}$, além de dispor de tamanho ajustado para filmes de mama (evitando áreas luminosas descobertas) e foco de luz lateral. Apesar disso, a lente de aumento não deve ser dispensada durante a leitura das imagens. Além disso, a luminosidade do ambiente não deve ser superior a 50 lux, para o que é necessário manter o local de leitura das imagens em penumbra. Deve-se evitar áreas do negatoscópio próximas ao filme "descobertas" durante a leitura das imagens, para que se evite a difusão de luz sobre o filme e a perda de detalhes. Para áreas muito escuras, um foco adicional deve ser disponível (em geral na lateral do negatoscópio específico), com luminância de no mínimo $20.000 \mathrm{~cd} / \mathrm{m}^{2}$. Segundo o protocolo inglês, a uniformidade da luminância deve ser de $\pm 15 \%{ }^{(8)}$. Nas normas nacionais, a tolerância aceita é de $\pm 20 \%$, tanto para uniformidade quanto para variações entre negatoscópios pró$\operatorname{ximos}^{(3)}$.

O estudo da detectabilidade clínica pode ser uma fonte de informação adicional ao programa de garantia de qualidade, pois permite identificar a sensitividade e a especificidade do método de diagnóstico. Este estudo já faz parte do programa de garantia de qualidade implementado desde 1999 no setor de Mastologia da Universidade Federal de São Paulo. O uso de simuladores estatísticos é de difícil aplicabilidade na rotina desses programas, mas pode ser útil quando se pretende avaliar um sistema gerador de imagem ou mesmo o próprio programa.

\section{CONCLUSÕES}

A detectabilidade é superior quando se utiliza o negatoscópio específico para ma- mografias, quando comparada à obtida com o negatoscópio "padrão”, para as imagens com densidades ópticas mais altas.

A detectabilidade é superior para $24 \mathrm{kV}$ (técnica de alto contraste) quando comparada a $28 \mathrm{kV}$ (AEC), para os dois tipos de negatoscópios utilizados.

As diferenças inerentes à subjetividade da interpretação tornam-se mais relevantes para filmes com densidades ópticas mais altas se interpretados em negatoscópios com luminância mais baixa.

A detectabilidade de fibras é superior à de microcalcificações, independentemente da luminância do negatoscópio.

\section{REFERÊNCIAS}

1. Gurvich VA. Statistical approach for image quality evaluation in daily medical practice. Med Phys 2000;27:94-100

2. Metz CE. Receiver operating characteristic (ROC) analysis in medical imaging. ICRU News, June 1997:7-16

3. Agência Nacional de Vigilância Sanitária do Ministério da Saúde. Regulamento técnico. Diretrizes de proteção radiológica em radiodiagnóstico médico e odontológico. 1/6/1998, no 453.

4. American College of Radiology. Committee on quality assurance in mammography. Mammography quality control: radiologist's manual. Merrifield, VA: ACR, 1992.

5. Institute of Physical Sciences in Medicine. Comissioning and routine testing of mammographic X-ray systems. Report no 59. York, England: IPSM, 1994.

6. Material técnico de divulgação interna da empresa Sylvania do Brasil.

7. Martinez LC, Rodriguez C, Gilarranz R, Muñoz C, Manzanas MJ. Niveles de luminosidad alcanzables en negatoscopios convencionales. (Resumen). Anais do XIII Congreso Nacional de Física Médica, Málaga, Espanha, 2001.

8. Medeiros RB, Alves FFR, Ferreira APG, Elias S, Fingerman F, Kemp C. How the qualiy control program can help the detectability of findings in mammograms. (Abstract). Anais do World Congress on Medical Physics and Biomedical Engineering, Chicago, 2000.

9. Haus AG, Yafee MJ. Syllabus: a categorical course in physics. Technical aspects of breast imaging. 3rd ed. Chicago: RSNA Publications, 1994.

10. Kimme-Smith C, Haus AG, DeBruhl N, Basset LW. Effects of ambient light and view box luminance on the detection of calcifications in mammography. AJR 1987;168:775-8. 